\title{
Téoros
}

Revue de recherche en tourisme

\section{Animal Subjects - An Ethical Reader in a Posthuman World, Jodey CASTRICANO (sous la direction de), Waterloo, Canada : Wilfrid Laurier University Press, 2008, 312 pages, ISBN-13 : 978-0-88920-512-3}

\section{Alain A. Grenier}

Volume 32, numéro 1, 2013

URI : https://id.erudit.org/iderudit/1036664ar

DOI : https://doi.org/10.7202/1036664ar

Aller au sommaire du numéro

Éditeur(s)

Université du Québec à Montréal

ISSN

0712-8657 (imprimé)

1923-2705 (numérique)

Découvrir la revue

Citer cette note

Grenier, A. A. (2013). Animal Subjects - An Ethical Reader in a Posthuman World, Jodey CASTRICANO (sous la direction de), Waterloo, Canada : Wilfrid Laurier University Press, 2008, 312 pages, ISBN-13 : 978-0-88920-512-3. Téoros, 32(1), 144-147. https://doi.org/10.7202/1036664ar d'utilisation que vous pouvez consulter en ligne. 


\section{POUR EN LIRE PLUS}

L'ouvrage regroupe, sous forme de chapitres, une collection de vingt textes traitant du concept et de l'historique de la chasse, de la combinaison de celle-ci au tourisme, des impacts de ce type de tourisme dit "de consommation» (consumptive wildlife tourism) et, enfin, des enjeux actuels liés à son développement. On y présente le niveau de complexité du sujet, la diversité des expériences recherchées par les touristes (la connexion avec les aliments, le contact avec la nature, les pratiques de cueillette traditionnelles ou technologiques), ainsi que les problèmes de gestion (conflits entre chasseurs ou avec des résidents chasseurs et non chasseurs, établissement de politiques, etc.).

Le livre éveille également le lecteur aux situations particulièrement problématiques des usages multiples du territoire, comme dans le cas de la pêche professionnelle ou de l'exploitation industrielle d'un lieu à des fins énergétiques. Les impacts du jumelage des activités de cueillette au tourisme sont nombreux et pas toujours positifs. L'ouvrage compare enfin les retombées économiques de la chasse à celles du simple tourisme d'observation de la faune et démontre comment les activités de chasse et de pêche peuvent aider les communautés à valoriser leur patrimoine naturel.

Si les exemples tirés aux quatre coins du monde offrent un éventail intéressant de la situation du tourisme de chasse et de pêche dans des contextes géoculturels relativement divers (du Nunavut à la Nouvelle-Zélande, en passant par l'Afrique), on reprochera néanmoins à l'éditeur de s'en être tenu au milieu anglo-saxon. De même, on regrettera que l'ouvrage ne se penche pas, à l'instar de notre dossier thématique, sur la notion du droit des animaux, autrement qu'à titre d'obstacle au développement des pratiques de chasse. Cette question, encore trop facilement évacuée, devra un jour ou l'autre être étudiée en profondeur. Dans la réalité, les positions mises de l'avant ne peuvent pas être toujours aussi extrêmes que la littérature nous le laisse croire.

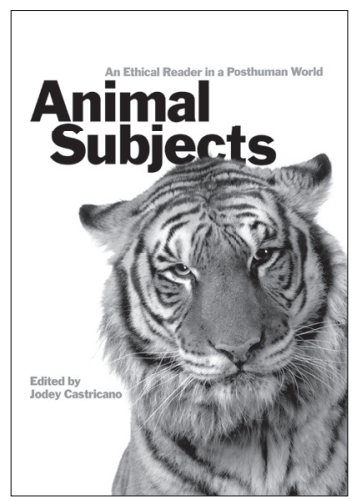

\section{Animal Subjects - An Ethical Reader in a Posthuman World}

Jodey CASTRICANO (sous la direction de)

Waterloo, Canada : Wilfrid Laurier University Press, 2008, 312 pages

ISBN-13 : 978-0-88920-512-3

L'éthique en matière de droits des animaux a longtemps été la « chasse gardée» de penseurs comme Tom Regan et Peter Singer. Bravant toutes les polémiques, leur courant de pensée a fait école et voilà qu'une nouvelle génération d'universitaires prend la relève. Sous la direction de Jodey Castricano de l'Université de la Colombie-Britannique en Okanagan, cet ouvrage réunit les travaux de treize chercheurs et chercheuses, qui posent un regard tout aussi critique sur le rapport entre les humains et le reste du monde animal.

Castricano et ses collègues s'attardent à la problématique entourant le débat sur la question du respect des animaux dans les pratiques et les études culturelles. Leur point de départ : une critique de la structure du monde actuel, dont ils dénoncent l'aspect hiérarchique d'un système organisé autour du genre, de l'ethnicité, du sexe et des classes sociales. Or, les études culturelles qui s'emploient notamment à démontrer ces constructions ont, selon les auteurs, exclu le monde animal. L'ouvrage vise donc à rectifier cet «oubli». (suite en page 147)

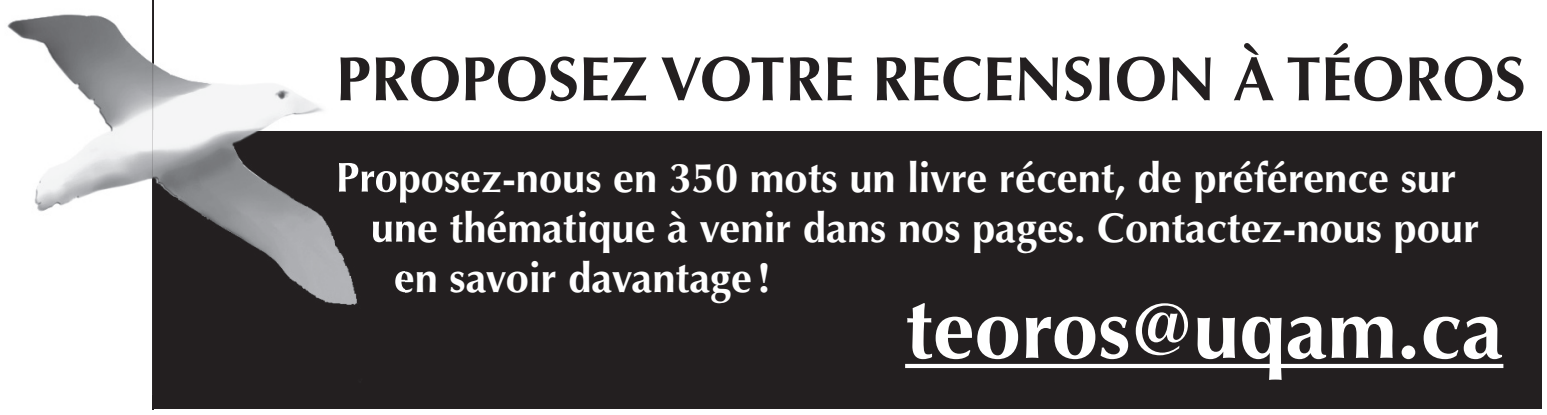


être parce que ces derniers sont moins photogéniques, et que d'autres espèces nous sont présentées comme étant nobles et dignes de notre protection. Ce raisonnement se situe dans le même registre que la dissonance cognitive qui permet aux gens d'avoir un chien ou un chat comme animal compagnie, mais d'en abattre d'autres, surtout quand ceux-ci ne sont pas considérés comme des individus.

\section{Le discours sur la protection et le respect de la faune est souvent orienté vers la protection des mammifères. Les poissons semblent avoir beaucoup moins attirer la sympathie du public. Devrait-on aussi se préoccuper des poissons?}

II est vrai que les gens ne croient pas que les poissons puissent ressentir de la douleur ou du plaisir. Toutefois, des études ont prouvé que cela est faux et que, en fait, non seulement les poissons ressentent la douleur et le plaisir, mais qu'ils ont aussi des vies émotionnelles complexes. Les gens ne partagent pas cette vision, peut-être parce qu'on ne considère pas que les poissons ont des expressions faciales. Cependant, les scientifiques ont démontré que les poissons rouges, par exemple, ont la capacité d'apprendre. II y a quelques années, nous appliquions la même forme de déni face aux poulets, mais la science a depuis révélé que ces oiseaux peuvent obtenir de meilleurs résultats que les chiens ou les chats lors de tests cognitifs. En d'autres mots, les poulets ont montré des niveaux de conscience de soi supérieurs à ceux qu'on leur attribuait traditionnellement. Pourtant, ils demeurent, comme les dindes, au nombre des animaux les plus mal traités par le secteur de l'élevage industriel. La façon dont les animaux sont traités dépend de notre perception à leur égard. Notre vision des dauphins et des baleines a évolué et pourrait bien, un jour, s'étendre aux poissons.

\section{Si la chasse est contraire à l'éthique, et si on ne peut pas devenir végétarien, quelles solutions avons-nous en tant que consommateurs?}

II existe un mythe selon lequel le mode de vie végétarien ne fournit pas suffisamment de protéines, mais environ 20 millions d'Américains sont végétariens ou végétaliens. Moins de $1 \%$ des animaux tués pour leur viande proviennent de petites fermes familiales. Les fermes industrielles modernes maintiennent généralement les vaches, les veaux, les cochons, les poulets, les dindes, les canards et autres dans des espaces surpeuplés, où ils ne peuvent même pas se retourner. Ces animaux sont, bien entendu, régulièrement soumis à de mauvais traitements et à des injections d'antibiotiques.

Tant d'animaux meurent afin que les gens puissent manger de la viande sous forme de hamburgers, de rôtis, de hot dogs, de bacon, mais tous ces termes cachent la réalité de l'abattage. Les protéines animales (y compris les produits laitiers) sont en fait liées aux maladies chroniques, aux maladies coronariennes et à certaines formes de cancer. Par exemple, deux études de l'université Harvard ont démontré que les hommes qui boivent du lait couraient $60 \%$ plus de risques de développer un cancer de la prostate que ceux qui évitent les produits laitiers. II est également important de reconnaitre que la consommation de protéines animales n'a jamais été une nécessité biologique, bien que l'on dise le contraire. En réalité, on mange de la viande parce que l'on nous a appris à le faire.
Quelles solutions avons-nous en tant que consommateurs? L'élevage d'animaux à des fins alimentaires se termine sur le plancher des abattoirs. Soyez conscient des activités que vous soutenez au nom du plaisir, alors que d'autres sources de protéines de meilleure qualité et plus respectueuses de l'environnement sont à votre disposition.

\section{Références}

CARTMILL, Matt (1996) A View to a Death in the Morning - Hunting and Nature Through History, Harvard University Press, 347 pages.

TAYLOR, Angus (2009) Animals and Ethics: An Overview of the Philosophical Debate, $3^{e}$ edition, Broadview Press, Peterborough, Ontario. 232 pages.

\section{POUR EN LIRE PLUS (suite de la page 144)}

Le livre, tantôt très universitaire, tantôt plus politique, s'adresse à un lectorat ouvert et prêt à revoir le modèle actuel des rapports quasi institutionnels avec les animaux. On y aborde autant des questions philosophiques, comme celles de la situation des animaux ("A Missed Opportunity: Humanism, Anti-Humanism and the Animal Question», Paola Cavalieri), de la nécessité de penser autrement («Thinking Other-Wise: Cognitive Science, Deconstruction and the (Non)Speaking (Non)Human Animal Subject", Cary Wolfe), du blâme et de la honte ("Blame and Shame? How Can We Reduce Unproductive Animal Experimentation?», Anne Innis Dagg), que des sujets plus pratiques, comme l'utilisation des animaux dans les domaines récréatif et touristique («Monsters: The Case of Marineland», John Sorenson).

À partir d'exemples et d'une recension élaborée d'ouvrages contemporains et plus anciens, Castricano et ses collègues retracent ici les origines des conventions qui régissent nos rapports avec les animaux. Ils démontrent comment, en les dénigrant à des rangs inférieurs, nous arrivons aujourd'hui à ne pas nous soucier de leur bien-être et à en abuser dans le cadre de nos activités quotidiennes, ou plus indirectement par nos choix de consommation, qui engendrent une exploitation à outrance du monde animal au nom de la supériorité humaine.

Alain A. Grenier, Ph.D., Département d'études urbaines et touristiques (ESG-UQAM) 